JOURNAL OF THEORETICAL

AND APPLIED MECHANICS

52, 4, pp. 981-993, Warsaw 2014

\title{
EXPERIMENTAL CONFIRMATION FOR THE VALIDITY OF RITZ METHOD IN STRUCTURAL DYNAMIC ANALYSIS
}

\author{
Gultekin Aktas, Abdulhalim Karasin \\ Dicle University, Engineering Faculty, Civil Engineering Department, 21280 Diyarbakır, Turkey \\ e-mail: gaktas@dicle.edu.tr; karasin@dicle.edu.tr
}

\begin{abstract}
The issue of Ritz vectors and their advantages is well-known in the field of structural engineering. This study deals with Ritz analysis and its experimental support. To that end, two different precast concrete molds were handled. Experiments were performed under vibration with the use of a computer-based data acquisition system. Transducers were used to measure displacement histories on the mold. Three dimensional finite element (3D FEM) simulation modeling of the concrete mold was carried out to obtain deflection histories on the mold. The computed displacement histories were compared with the experimental testing results. The comparisons show that the experimental and numerical results are compatible.
\end{abstract}

Keywords: experimental study, concrete mold, Ritz vectors, 3D FEM, mode-superposition method

\section{Introduction}

The computation time and mass participation ratios are two significant parameters for large structures that have too many degrees of freedom (DOF). Eigenvector analysis determines undamped free-vibration mode shapes and frequencies of the system. These natural modes provide an excellent insight into the behavior of the structure. Ritz-vector analysis finds modes that are excited by a particular loading. Ritz vectors can provide a better basis than the eigenvectors do when used for response-spectrum or time-history analyses that are based on modal superposition. The engineers may determine the type of modes which they feel are the most appropriate.

It has usually been assumed that the best possible basis for reducing the size of a system to perform a mode superposition analysis is to use the exact eigenvectors of the total system. Ritz and component mode synthesis methods have been used extensively for the dynamic analysis of many practical structures. However, the major motivation for their use has been to avoid the large eigenvalue problem rather than to obtain more accurate results (Guyan, 1965; Dickens and Wilson, 1980).

The participation of particular eigenvectors in the final solution will depend on the properties of the dynamic loading. It is well-known that a mode shape with a natural frequency near the dominant frequencies of the loading significantly participates in the solution. However, the spatial distribution of the loading is of equal importance. Eigenvectors, which are orthogonal to the loading, are not excited even if their frequency is involved in the loading. Also, for concentrated loads, a large number of eigenvectors can be required to capture the static load effects (Schendler and MacNeal, 1962; Hansteen, 1979; Powell, 1979).

Some studies have indicated that the natural free-vibration mode shapes are not the best basis for a mode-superposition analysis of structures subjected to dynamic loads. Wilson et al. (1982) demonstrated that dynamic analyses based on a special set of load-dependent Ritz vectors yield more accurate results than the use of the same number of natural mode shapes. The reason why the Ritz vectors yield excellent results is that they are generated by taking into consideration the spatial distribution of the dynamic loading, whereas the direct use of the 
natural mode shapes neglects this very important information. Since the Ritz-vector algorithm is faster than the eigenvector algorithm, the former is recommended for time-history analyses. They also reported that the solution to the eigenvalue problem for large structures is often the most costly phase of the dynamic response analysis.

Ritz vectors were used by Dong et al. (2001) to reduce the size of the matrices required for the analysis. Numerical simulations verified the effectiveness and accuracy of the Ritz vector method.

In literature, there are some numerical studies performed making use of the finite element method (FEM) and/or Ritz vectors-eigenvectors to compute the behavior of structural members (Karasin and Gulkan, 2007; Zhou and Zheng, 2008; Cunedioglu, 2011; Jung et al., 2011; Özütok and Madenci, 2013; Mazurkiewicz et al., 2013).

The main purpose of this study is to provide experimental contributions to the validity of the Ritz vector method in terms of the application to structural systems. To this end, a simulation method of the behavior of a steel mold was performed for two precast concrete structures when they were subjected to vibration by an external vibrator. The numerical model of the mold was carried out by using the three dimensional finite element method (3D FEM). Time-history analysis was performed by the mode superposition method, in which load-dependent Ritz vectors and undamped free-vibration modes (eigenvectors) were used alternatively. In this study, the experimental results for mold deflection histories under vibration load obtained by Aktas et al. (2014) are compared with the numerical results. Moreover, the Ritz vectors results are compared with those of eigenvectors.

\section{Experimental study}

In the content of current study, two full scale molds of precast concrete elements having geometry of real sizes were utilized. The views of the two different molds (Box culvert and Column element) used in the experiments are illustrated in Figs. 1 to 4.

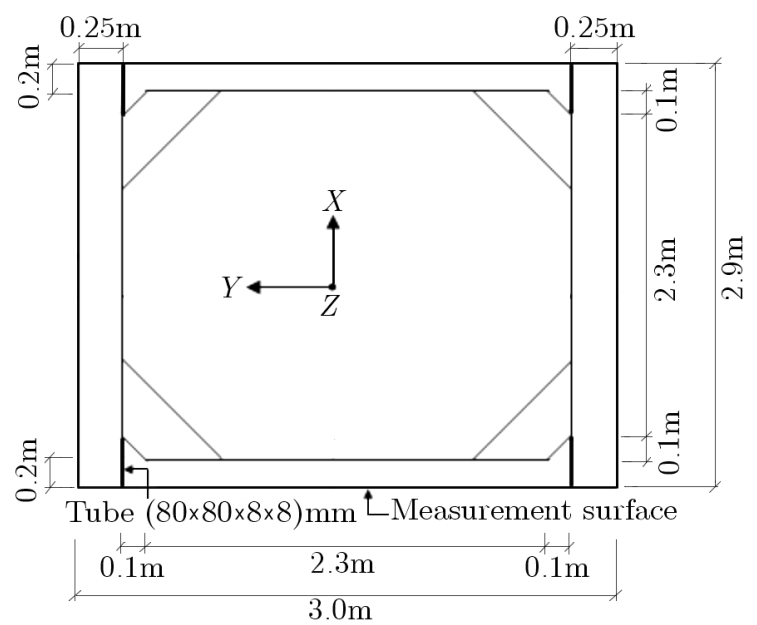

Fig. 1. Plan view of the Box culvert mold $(h=0.97 \mathrm{~m})$

1 and 2 points are measurement points in Fig. 3, and $A, B$ and $C$ points are measurement points in Fig. 4. The length of Column mold is $10.29 \mathrm{~m}$, and the size of Box culvert mold is $3.0 \times 2.9 \mathrm{~m}$. Using these molds, manufacturing is made for real engineering applications in the precast concrete production workshop. The molds used for test specimens are made of steel plates of $5 \mathrm{~mm}$ thickness. Steel profiles in various size and sections are connected to the molds in horizontal, vertical and diagonal directions in order to strengthen the system. 


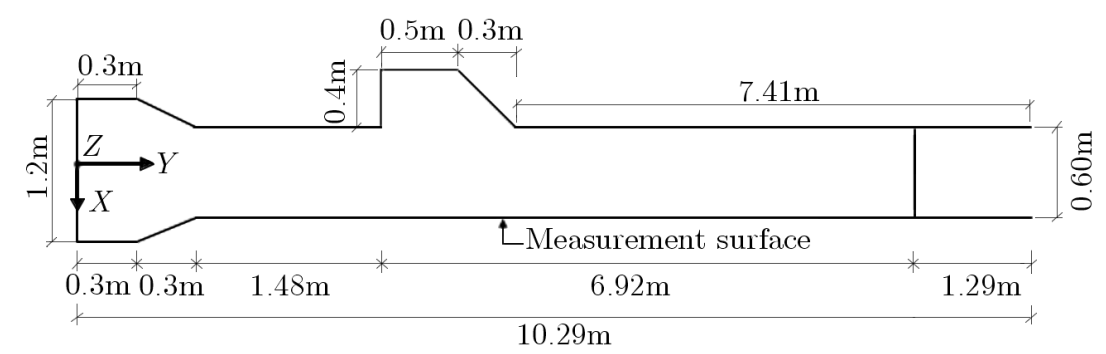

Fig. 2. Plan view of Column mold $(h=0.60 \mathrm{~m})$

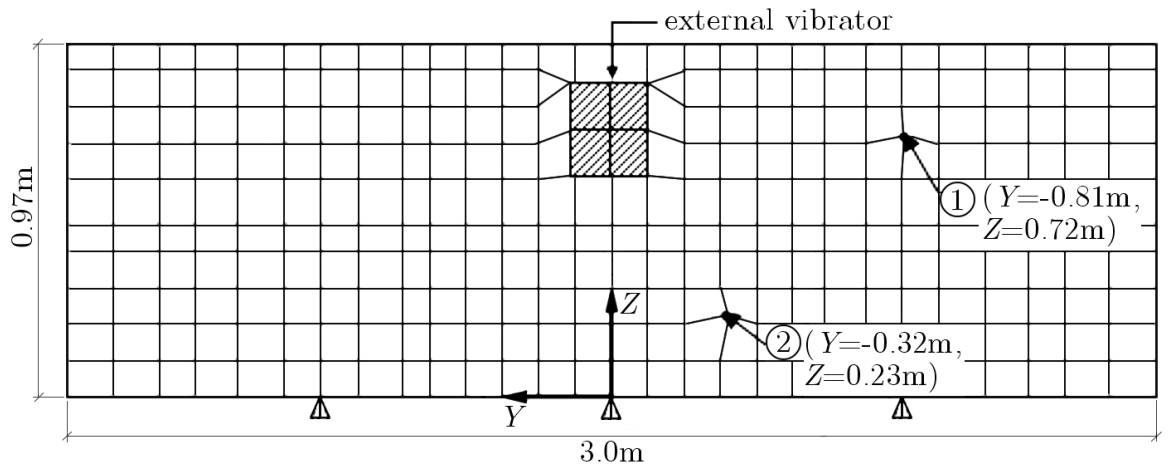

Fig. 3. Measurement surface of the finite element mesh of Box culvert mold ( $Y Z$-plane, $X=-1.45 \mathrm{~m}$ )

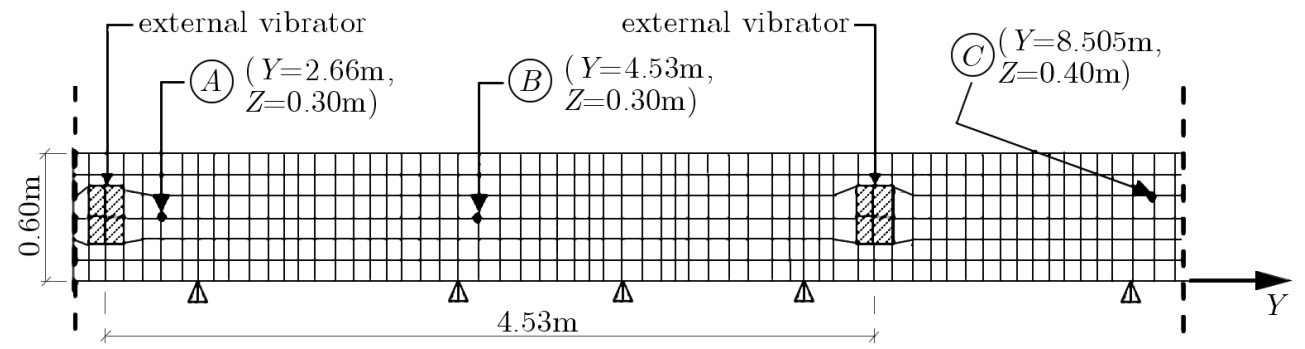

Fig. 4. Measurement surface of the finite element mesh of Column mold ( $Y Z$-plane, $X=0.30 \mathrm{~m}$ )

The external vibrators used in the experiments are clamped on a rigid steel plate having dimensions of $200 \times 250 \mathrm{~mm}$. The specifications of the external vibrator are presented in Table 1 .

The experimental measurements were taken on the surface of the molds for two different precast concrete structural units by using a computer-based data acquisition system. The location of measurement points were selected near and far from vibration points to see their differences and effect. The data acquisition system used in this study, including instrumentation, hardware and software, is described as follows.

\subsection{Instrumentation}

The calibration of the displacement transducer was carried out using a dial gauge with a sensitivity of $0.01 \mathrm{~mm}$ (Fig. 5). Displacements measured by the transducers were recorded simultaneously with $0.5 \mathrm{~ms}$ (millisecond) of the sampling rate for a duration of $4.096 \mathrm{~s}$. Sampling rates were determined so that sinusoidal function, Eq. $(3.2)_{1}$ reaches the maximum value. $0.5 \mathrm{~ms}$ $(0.1 \pi)$ sampling rate reaches the maximum value in 5 th $(0.5 \pi)$ and 15 th $(1.5 \pi)$ step in the sinusoidal function. The displacement amplitude was reached to the maximum level in less than a second duration.

Although the acronym stands for Linear Variable Differential Transformer, an LVDT is often referred to as a linear displacement transducer that measures linear displacement. There 
Table 1. Specifications of the external vibrator

\begin{tabular}{|c|c|c|c|c|c|c|}
\hline \multicolumn{5}{|c|}{ Mechanical features } & \multicolumn{2}{c|}{ Electrical features } \\
\hline Vibration range & \multicolumn{2}{|c|}{ Centrifugal force } & Weight & Max. input power & \multicolumn{2}{c|}{ Max. current $A$} \\
\hline$[$ Vibr./min] & {$[\mathrm{kg}]$} & {$[\mathrm{kN}]$} & {$[\mathrm{kg}]$} & {$[\mathrm{W}]$} & $42 \mathrm{~V}$ & $250 \mathrm{~V}$ \\
\hline \hline $6000(200 \mathrm{~Hz})$ & 1157 & 11.34 & 25 & 1200 & 23 & - \\
\hline
\end{tabular}

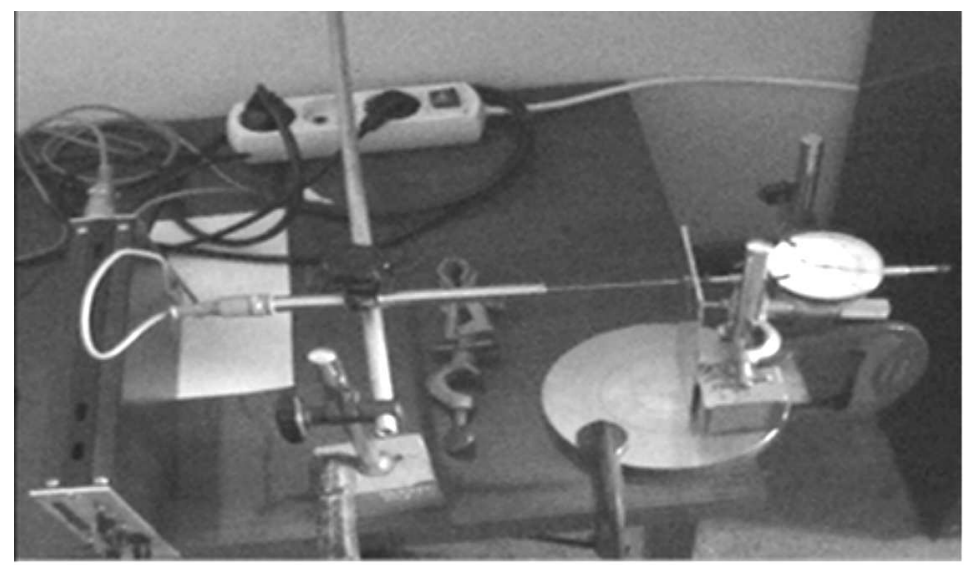

Fig. 5. Calibration setup of the displacement transducer

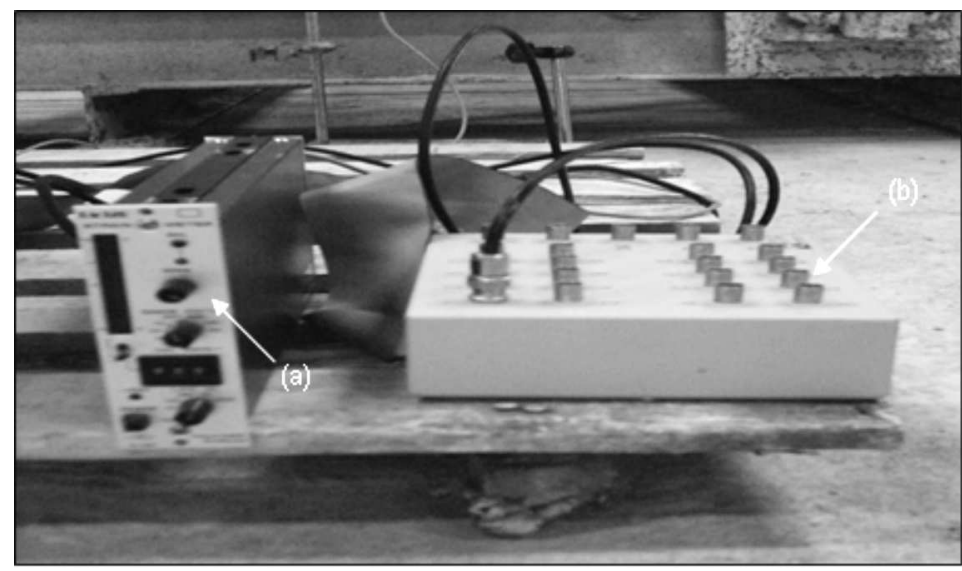

Fig. 6. (a) Dynamic strain meter; and (b) Junction-box

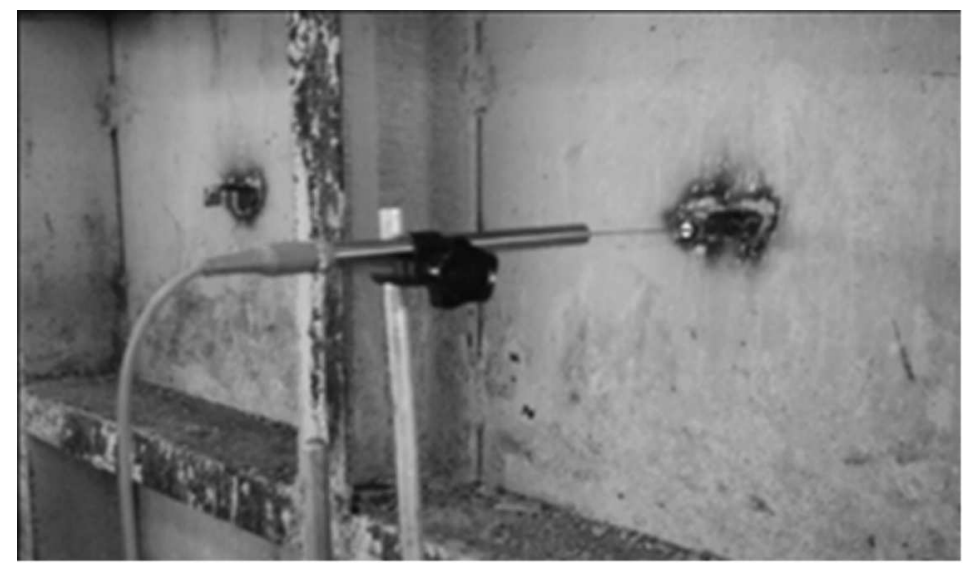

Fig. 7. LVDT connection of the mold 
are some experimental works made utilizing LVDTs to measure displacements in structural elements (Muller-Rochholz et al., 1986; Oh and Kim, 2004; Lee and Pessiki, 2008; Iskander et al., 2012).

In this study, the following hardware and software was used: 1 - dynamic strain meter (Fig. 6); 2 - LVDT (displacement transducer); and 3 - data acquisition card. Software: SIGNALYS Program (Ziegler-Instruments GmbH, Germany, 1990). LVDT connection of the mold is given in Fig. 7.

\section{The mode superposition method}

The standard mode-superposition method of response analysis is used to solve the dynamic equilibrium equations of motion for the complete structure. The modes used in the analysis can be undamped free-vibration modes (eigenvectors) or load-dependent Ritz vector modes alternatively.

The dynamic equilibrium equation of a structural system, modeled by a finite number of discrete members and lumped masses, is written in terms of joint displacements as

$$
\mathbf{M a}(t)+\mathbf{C v}(t)+\mathbf{K u}(t)=\mathbf{r}(s, t)
$$

where $\mathbf{M}, \mathbf{C}$ and $\mathbf{K}$ are given $N \times N$ mass, damping and stiffness matrices, respectively. $N$ is the number of degrees of freedom of the system. The time-dependent vectors $\mathbf{a}, \mathbf{v}$ and $\mathbf{u}$ are the joint accelerations, velocities and displacements, respectively; $\mathbf{r}$ is the arbitrary applied load, varying with the position $s$ and time $t$.

The arbitrary loading in Eq. (3.1) will be harmonic if the structure is loaded by external vibrators, and can be written as

$$
\mathbf{r}(s, t)=\mathbf{f}_{o} \sin (\omega t) \quad \omega=2 \pi f \quad T=\frac{2 \pi}{\omega}
$$

in which $f_{o}, \omega$ and $f$ are constant centrifugal force (the amplitude of the load), angular and cyclic frequency of the vibrator, respectively; $\sin (\omega t)$ is the function of time, and $T[\mathrm{~s}]$ is the period. It should be noted that the load applied by the vibrator is perpendicular to the surface of the mold.

Wilson et al. (1982) stated that the mode superposition method is based on displacement transformation given below

$$
\mathbf{u}(s, t)=\mathbf{X}(s) \mathbf{u}_{x}(t) \quad \text { or } \quad \mathbf{u}=\mathbf{X} \mathbf{u}_{x}
$$

where $\mathbf{u}_{x}$ is an array of $L$ unknown time functions and $\mathbf{X}$ is an $N \times L$ matrix of shape functions. The corresponding velocities and accelerations are

$$
\mathbf{v}=\mathbf{X} \mathbf{v}_{x} \quad \mathbf{a}=\mathbf{X} \mathbf{a}_{x}
$$

The substitution of equations (3.3) and (3.4) into equation (3.1) and pre-multiplication by $\mathbf{X}^{\mathrm{T}}$ gives

$$
\mathbf{M}^{*} \mathbf{a}_{x}+\mathbf{C}^{*} \mathbf{v}_{x}+\mathbf{K}^{*} \mathbf{u}_{x}=\mathbf{f}^{*}(s) g(t)
$$

in which

$$
\mathbf{M}^{*}=\mathbf{X}^{\mathrm{T}} \mathbf{M X} \quad \mathbf{C}^{*}=\mathbf{X}^{\mathrm{T}} \mathbf{C X} \quad \mathbf{K}^{*}=\mathbf{X}^{\mathrm{T}} \mathbf{K X} \quad \mathbf{f}^{*}=\mathbf{X}^{\mathrm{T}} \mathbf{f}
$$

where $\mathbf{X}$ is a matrix of the lowest eigenvectors of the system, the matrices $\mathbf{M}^{*}$ and $\mathbf{K}^{*}$ are diagonal for the traditional mode superposition method, and the modal matrix $\mathbf{C}^{*}$ is assumed 
also diagonal. Thus, Eq. (3.5) is reduced to a set of uncouples, linear, second-order, ordinary differential equations. Each equation can be solved for the unknown time function by any of several standard methods (Dickens and Wilson, 1980). On the other hand, if $\mathbf{X}$ is a matrix of Ritz vectors, the $L \times L$ matrices are not diagonal. The solution to this coupled set of equations can be obtained with a minimum of numerical effort since $L$ is always small compared to the size of the complete system $N$.

\subsection{Assessment of Ritz vectors}

Wilson et al. (1982) defined that the selection of Ritz vectors is an extremely critical phase if they are to be used directly in a superposition analysis. The vectors are generated from the following recurrence relationship

$$
\mathbf{K x}_{i}^{*}=\mathbf{M x}_{i-1} \quad i=2, \ldots, N
$$

The first vector is obtained from the solution

$$
\mathbf{K} \mathbf{x}_{i}^{*}=\mathbf{f}
$$

The vectors are orthogonalized and normalized at each step by the use of the following procedure

$$
\mathbf{x}_{i}=\mathbf{x}_{i}^{*}-\sum_{j=1}^{i-1} c_{j} \mathbf{x}_{j}
$$

where $c_{j}=\mathbf{x}_{j}^{\mathrm{T}} \mathbf{M} \mathbf{x}_{i}^{*}$, since after each vector is found, it is normalized as

$$
\mathbf{x}_{j}^{\mathrm{T}} \mathbf{M} \mathbf{x}_{j}=1
$$

The first Ritz vector represents a static response to the load vector $\mathbf{f}$. The dynamic forces which are neglected in this static analysis have the form $\omega^{2} \mathbf{M} \mathbf{x}_{1}$, where $\omega$ represents a typical frequency component of the loading. This error vector is then applied in the next step as a load in order to generate the next Ritz vector. Again, this static solution neglects dynamic terms which are used as the next load vector for the generation of the next Ritz vector. This sequence of steps will terminate when the next vector generated is orthogonal to all the vectors found previously. The detailed evaluation of Ritz vectors can be seen in Wilson et al. (1982).

\section{Three-dimensional modeling of molds}

The full scale molds are made of steel plates of $5 \mathrm{~mm}$ thickness. Steel profiles in various size and sections are connected to the molds as horizontal, vertical and diagonal in order to strengthen the system. Reinforcing steel profiles located at the bottom of the mold are assumed to be simply supported in the model. Boundary conditions of the molds for Box culvert and Column elements are given in Table 2 .

The finite element mesh of the molds is formed with Frame and Shell elements. The shell finite elements are formed nearly in dimensions of $100 \times 100 \mathrm{~mm}$ as quadrilateral, defined by four joints as square, rectangular or trapezoidal elements; and triangular, defined by three joints, as transition aimed triangular elements depending on the mold geometry. The frame elements are associated with the Shell elements by defining them on the same joints.

The two and three-dimensional views of the molds obtained from 3D FEM are illustrated in Figs. 3 and 8 for Box culvert mold, and in Figs. 4 and 9 for Column mold. 
Table 2. The boundary conditions of mold systems. The coordinates of joints where $u=0$ $(X Y$-plane, $Z=0)$

\begin{tabular}{|c|c|c|c|}
\hline \multicolumn{2}{|c|}{ Box culvert mold } & \multicolumn{2}{c|}{ Column mold } \\
\hline$X[\mathrm{~mm}]$ & $Y[\mathrm{~mm}]$ & $X[\mathrm{~mm}]$ & $Y[\mathrm{~mm}]$ \\
\hline \hline-750 & -1500 & 600 & 100 \\
\hline-50 & -1500 & 300 & 720 \\
\hline 750 & -1500 & 300 & 1260 \\
\hline-750 & -1250 & 300 & 1820 \\
\hline-50 & -1250 & 300 & 2880 \\
\hline 750 & -1250 & 300 & 4410 \\
\hline 1450 & -800 & 300 & 5370 \\
\hline 1450 & 0 & 300 & 6440 \\
\hline 1450 & 800 & 300 & 8370 \\
\hline 1250 & -800 & 300 & 10080 \\
\hline 1250 & 0 & -600 & 100 \\
\hline 1250 & 800 & -300 & 720 \\
\hline 750 & 1500 & -300 & 1260 \\
\hline-50 & 1500 & -300 & 1820 \\
\hline-750 & 1500 & -300 & 2880 \\
\hline 750 & 1250 & -300 & 4410 \\
\hline-50 & 1250 & -300 & 5370 \\
\hline-750 & 1250 & -300 & 6440 \\
\hline-1450 & 800 & -300 & 8370 \\
\hline-1450 & 0 & -300 & 10080 \\
\hline-1450 & -800 & & \\
\hline-1250 & 800 & & \\
\hline-1250 & 0 & & \\
\hline-1250 & -800 & & \\
\hline
\end{tabular}

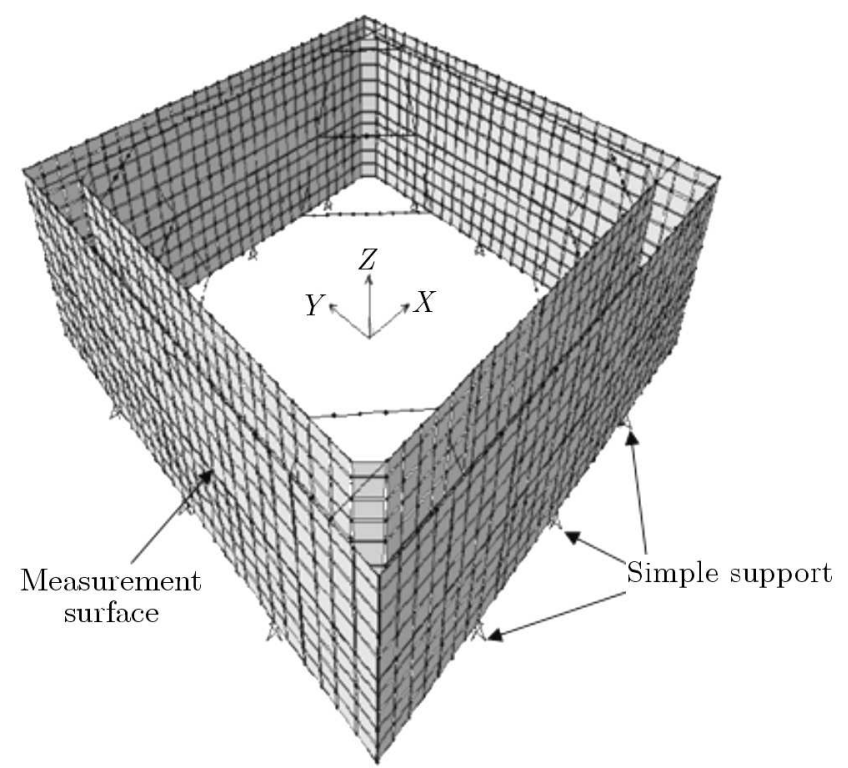

Fig. 8. Three-dimensional view of the finite element mesh for Box culvert mold 


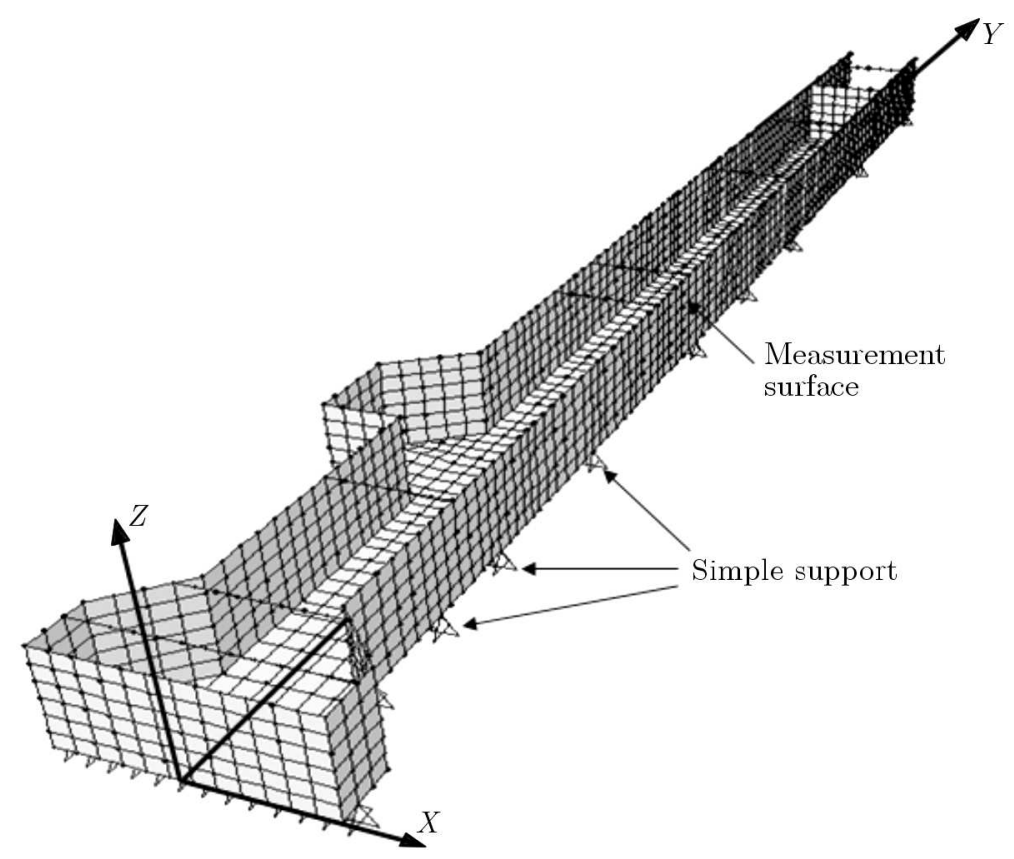

Fig. 9. Three-dimensional view of the finite element mesh for Column mold

It is assumed that the motion of the mold starts from the rest, and then the initial conditions for the mold are

$$
u(t=0)=0 \quad v(t=0)=0
$$

The numerical solution of Eq. (3.1) was carried out by using structural analysis and design software called SAP2000® (1999).

\section{Case studies}

In the applications, the mold systems of Box culvert and Column elements are taken into consideration. The frequency of the external vibrator used in experiments is $100 \mathrm{~Hz}$ (Wenzel, 1986).

The material properties of the steel mold taken by software are as follows: Young's modulus $E=199948 \mathrm{~N} / \mathrm{mm}^{2}$; specific weight $\gamma=7.682 \cdot 10^{-5} \mathrm{~N} / \mathrm{mm}^{3}$; Poisson's ratio $\nu=0.3$.

Displacement histories are in the normal direction to the surface of the mold. In the figures, the comparisons are illustrated in the steady state part of the behavior. Although some irregularities in the behavior are indicated in the earlier time interval, they disappear shortly and the behavior becomes uniform. Thus, the graphics are represented for a typical time period where the behavior is uniform.

In this Section, 3D FEM results are compared with the experimental ones. In the numerical analysis, the damping ratio is assumed as $5 \%$ for all modes in accordance with Turkish Earthquake Code (TEC, 2007). The frequency of the vibrator, the time step, number of time steps, and time span in the analyses are selected respectively as $100 \mathrm{~Hz}, 0.5 \mathrm{~ms}, 8192 \mathrm{~s}$ and $4.096 \mathrm{~s}$, which are the same as in the experimental records (Aktas et al., 2014). Computations of the joint displacement have been carried out at two measurement points named 1 and 2 (see Fig. 3) for the Box culvert mold, and at three measurement points named $A, B$ and $C$ (see Fig. 4) for the Column mold. The applications are presented as follows. 


\subsection{Exercise 1}

In this exercise, free vibration analysis of the mold systems for the Box culvert and Column elements is performed. The cyclic frequencies of the first six undamped free vibration modes are given in Table 3.

Table 3. Free-vibration frequencies of the mold systems

\begin{tabular}{|c|c|c|}
\hline \multirow{2}{*}{ Modes } & \multicolumn{2}{|c|}{ Frequency $[\mathrm{Hz}]$} \\
\cline { 2 - 3 } & Box culvert mold & Column mold \\
\hline \hline 1 & 31.64 & 8.69 \\
\hline 2 & 41.90 & 12.42 \\
\hline 3 & 41.99 & 15.23 \\
\hline 4 & 45.65 & 16.54 \\
\hline 5 & 49.00 & 21.59 \\
\hline 6 & 50.89 & 39.37 \\
\hline
\end{tabular}

As seen in Table 3, the most effective frequencies of the systems are far away from the cyclic frequency of $100 \mathrm{~Hz}$. Hence, the resonance situation does not occur in the systems.

\subsection{Exercise 2}

In this case, computations of joint displacement histories of the molds have been carried out and compared with the experimental results. Comparison of time histories is made at two measurement points named 1 and 2 using one vibrator (see Fig. 3) for the Box culvert mold and at three measurement points named $A, B$ and $C$ using two vibrators (see Fig. 4) for the Column mold. The time-history analysis of the mold system has been executed using 80 Ritz modes. The numerical results are compared with the experimental ones in Figs. 10 to 14 for a typical time period. Figures 10 and 11 are given for the Box culvert mold as follows.

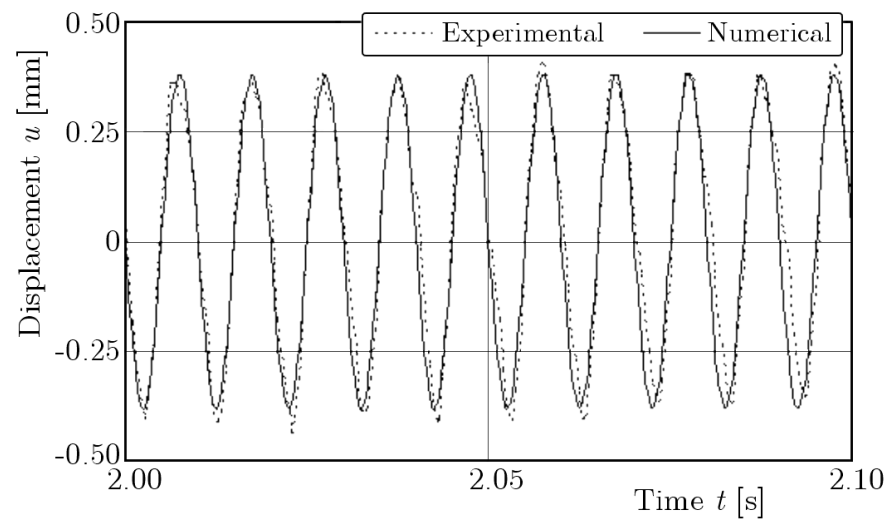

Fig. 10. Numerical and experimental displacement histories of the Box culvert mold (point 1)

Figures 12 to 14 are given for the Column mold as follows.

The number of mass degrees of freedom is 7353 and 6330 for the Box culvert and Column molds, respectively. Eigenvalue and Ritz vector analyses of the mold systems have been performed alternatively by using in various numbers of modes.

The modal mass participation ratios obtained from the analyses are compared in Table 4 and 5 .

Tables 4 and 5 demonstrate that the mass participation ratios obtained by using Ritz vectors are much greater than those of eigenvectors. 


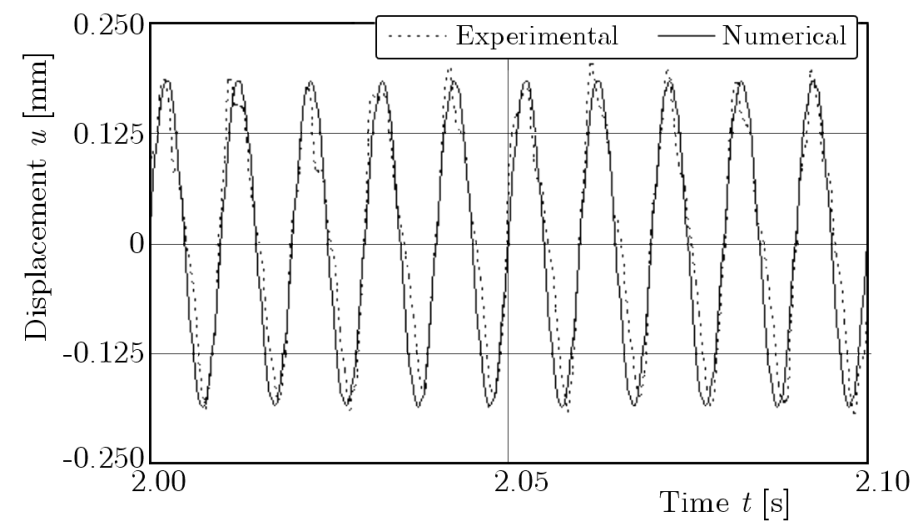

Fig. 11. Numerical and experimental displacement histories of the Box culvert mold (point 2)

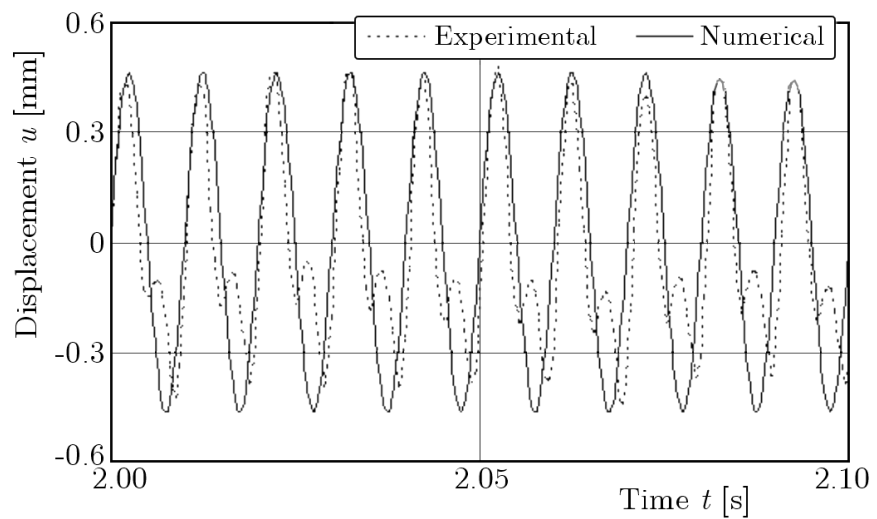

Fig. 12. Numerical and experimental displacement histories of the Column mold (point $A$ )

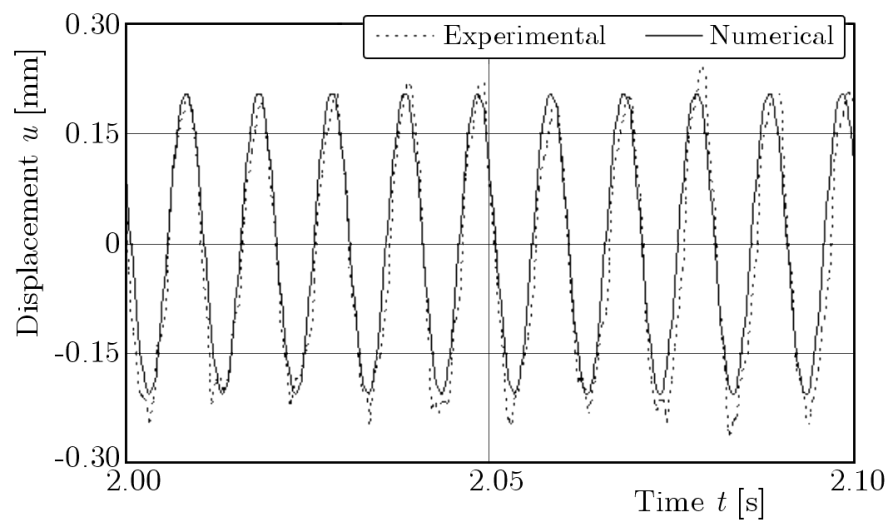

Fig. 13. Numerical and experimental displacement histories of the Column mold (point B)

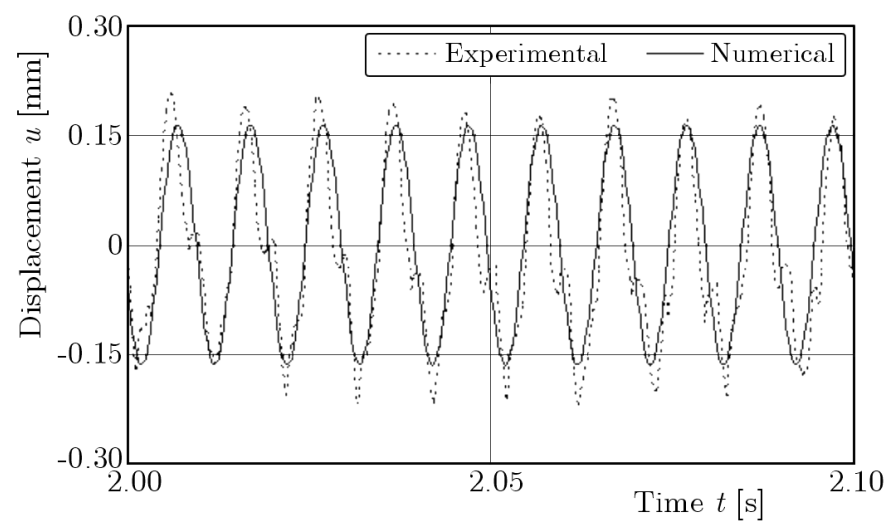

Fig. 14. Numerical and experimental displacement histories of the Column mold (point $C$ ) 
Table 4. Modal mass participation ratios of the Box culvert mold

\begin{tabular}{|c|c|c|c|c|}
\hline \multirow{2}{*}{$\begin{array}{c}\text { Number of } \\
\text { modes }\end{array}$} & Vector & \multicolumn{3}{|c|}{ Modal mass participation ratios in direction [\%] } \\
\cline { 3 - 5 } & type & $X$ & $Y$ & $Z$ \\
\hline \hline \multirow{2}{*}{50} & Eigenvectors & 27.81 & 28.94 & 0.01 \\
\cline { 2 - 5 } & Ritz vectors & 92.53 & 93.48 & 98.84 \\
\hline \multirow{2}{*}{100} & Eigenvectors & 35.01 & 31.44 & 0.02 \\
\cline { 2 - 5 } & Ritz vectors & 98.30 & 98.08 & 99.63 \\
\hline \multirow{2}{*}{150} & Eigenvectors & 52.35 & 50.66 & 0.18 \\
\cline { 2 - 5 } & Ritz vectors & 99.37 & 99.29 & 99.84 \\
\hline \multirow{2}{*}{250} & Eigenvectors & 59.84 & 59.89 & 5.50 \\
\cline { 2 - 5 } & Ritz vectors & 99.81 & 99.82 & 99.95 \\
\hline \multirow{2}{*}{500} & Eigenvectors & 69.42 & 73.74 & 86.95 \\
\cline { 2 - 5 } & Ritz vectors & $\cong 100$ & $\cong 100$ & $\cong 100$ \\
\hline \multirow{2}{*}{650} & Eigenvectors & 80.68 & 81.09 & 92.86 \\
\cline { 2 - 5 } & Ritz vectors & $\cong 100$ & $\cong 100$ & $\cong 100$ \\
\hline
\end{tabular}

Table 5. Modal mass participation ratios of the Column mold

\begin{tabular}{|c|c|c|c|c|}
\hline \multirow{2}{*}{$\begin{array}{c}\text { Number of } \\
\text { modes }\end{array}$} & \multirow{2}{*}{$\begin{array}{c}\text { Vector } \\
\text { type }\end{array}$} & \multicolumn{3}{|c|}{ Modal mass participation ratios in direction [\%] } \\
\cline { 3 - 5 } & E & $X$ & $Y$ & $Z$ \\
\hline \hline \multirow{2}{*}{50} & Eigenvectors & 72.00 & 1.36 & 4.51 \\
\cline { 2 - 5 } & Ritz vectors & 94.12 & 89.94 & 96.89 \\
\hline \multirow{2}{*}{100} & Eigenvectors & 74.46 & 2.49 & 29.42 \\
\cline { 2 - 5 } & Ritz vectors & 98.50 & 95.67 & 99.40 \\
\hline \multirow{2}{*}{150} & Eigenvectors & 76.10 & 4.67 & 37.14 \\
\cline { 2 - 5 } & Ritz vectors & 99.51 & 98.20 & 99.77 \\
\hline
\end{tabular}

\section{Results and discussion}

The aim of this Section is to examine, discuss and compare the results obtained from the experiments and FEM model. The experimental measurements were taken on the full scale molds for two different precast concrete elements by using a computer-based data acquisition system. Displacement histories were measured from the surface of the mold of these members, while the mold is empty (without fresh concrete in it). Displacements measured by the transducers were recorded simultaneously with $0.5 \mathrm{~ms}$ of the sampling rate for a duration of $4.096 \mathrm{~s}$. Sampling rates were determined so that the sinusoidal function, Eq. $(3.2)_{1}$, reaches the maximum value. As previously mentioned, the developed FEM models were tested with experimental data.

The standard mode-superposition method of response analysis was used to solve the dynamic equilibrium equations of motion for the complete structure. The modes used in the analysis can be undamped free-vibration modes (eigenvectors) or load-dependent Ritz vector modes of the system, alternatively.

Time-history analysis was performed by the mode-superposition method that utilizes load-dependent Ritz vectors or eigenvectors. The results found by using Ritz vectors were compared with those of eigenvectors.

As seen in Table 4, the use of only fifty Ritz modes in the analysis is sufficient to pass mass participation ratios of $90 \%$ in the global $X, Y$ and $Z$ directions. At the same time, in the same model, even using 650 eigen modes, does not exceed mass participation ratios of $90 \%$ in the global $X, Y$ directions. 
As stated previously, one vibrator was used on the Box culvert mold and two vibrator on the Column mold. The disturbances of the Column mold are larger than those of the Box culvert mold (see Figs. 10 to 14). The reason for this should be the use of two vibrators on the column mold. The largest disturbance occurred at measurement point $A$ of the Column mold (see Fig. 12). The first reason for the disturbances may be the phase difference (the moment to start operation) occurring between the two vibrators. The second reason may be that point $A$ is near to the vibrator. The third reason may be non-uniformity of the mold behavior along the mold surface. A more important issue in this study is that the experimental and numerical extreme amplitudes should be compatible with each other.

\section{Conclusions}

In the content of numerical study, the modeling of molds used in experiments has been accomplished by the three-dimensional finite element method (3D FEM) using SAP2000® software. The computed displacement amplitude histories are compared with the experimental ones with the aim of verification of the adequacy of the FEM model. The comparisons show that the measured and the computed results are compatible with each other (see Figs. 10 to 14) when Ritz vectors are used in the mode-superposition method.

Time-history analysis has been performed by the mode-superposition method that utilizes load-dependent Ritz vectors or eigenvectors. The results found by using Ritz vectors are compared with those of eigenvectors. In numerical analyses, the use of Ritz vectors in any number of modes not only significantly reduces computer time but also provides greater mass participation ratios when compared to eigenvectors (see Tables 4 and 5), provided that the same number of modes are used. Consequently, for complex structural systems that have the mass degrees of freedom of several thousands, it is recommended to use Ritz vectors in the mode superposition method in the dynamic analysis.

\section{References}

1. Aktas G., Tanrikulu A.K., Baran T., 2014, Computer-aided mold design algorithm for precast concrete elements, ACI Materials Journal, 111, 1, 77-87

2. Cunedioglu Y., 2011, Analyses of laminated cantilever composite beams by model order reduction techniques, Mechanics Based Design of Structures and Machines, 39, 22-45

3. Dickens J.M., Wilson E.L., 1980, Numerical Method for Dynamic Substructure Analysis, UCB/EERC- $80 / 20$

4. Dong Q., Matsui K., Yamamoto K., 2001, Time domain back calculation of pavement structure material properties using 3D FEM with Ritz vectors, International Journal of Geomechanics, 1, $325-336$

5. GuYAn R.J., 1965, Reduction of stiffness and mass matrices, AIAA Journal, 3, 380

6. HANSTEEN O.E., 1979, On the accuracy of mode superposition analysis in structural dynamics, Earthquake Engineering and Structural Dynamics, 7, 405-411

7. Iskander M., Parikh S., Aboumoussa W., 2012, Apparent thermal coefficient of expansion of concrete building with restraint, ACI Materials Journal, 109, 1, 63-70

8. Jung N., Patera A.T., Haasdonk B., Lohmann B., 2011, Model order reduction and error estimation with an application to the parameter-dependent eddy current equation, Mathematical and Computer Modelling of Dynamical Systems, 17, 561-582

9. Karasin A., GulKan P., 2007, Finite grid analogy for levy plates on generalized foundations, Turkish Journal of Engineering and Environmental Sciences, 31, 157-170 
10. LeE B., Pessiki S., 2008, Experimental evaluation of precast, prestressed concrete, three-wythe sandwich wall panels, PCI Journal, 53, 2, 1-21

11. Mazurkiewicz L., Mąachowski J., Baranowski P., Damaziak K., 2013, Comparison of numerical testing methods in terms of impulse loading applied to structural elements, Journal of Theoretical and Applied Mechanics, 51, 3, 615-25

12. Muller-Rochholz J.F.W., Fiebrich M., Breitbach M., 1986, Measurement of horizontal bridge movements due to temperature, wind, and traffic loadings, ACI Special Publication, 94, 409-418

13. Он B.H., Kiм K.S., 2004, Shear behavior of full-scale post-tensioned prestressed concrete bridge girders, ACI Structural Journal, 101, 2, 176-182

14. Özütok A., MAdenci E., 2013, Free vibration analysis of cross-ply laminated composite beams by mixed finite element formulation, International Journal of Structural Stability and Dynamics, 13

15. Powell G.H., 1979, Missing Nass Correction in Modal Analysis of Piping System, SMIRT Conf., Berlin

16. SAP2000®, 1999, Integrated finite element analysis and design of structures, Computers and Structures, Inc., Berkeley, California, USA

17. Schendler R.G., MacNeal R.H., 1962, Optimum structural representation in aeroelastic analysis, Aeronautical Systems Division, WPAEB, Ohio, U.S.A., ASD-TR-61-680

18. TEC Turkish Earthquake Code, 2007, Ministry of Public Works and Settlement, Specification for Structures to be Built in Disaster Areas, Government of Republic of Turkey

19. Wenzel D., 1986, Compaction of concrete - principles, practice, special problems, Betonwerk + Fertigteil Technic, 52, 3, 153-158

20. Wilson E.L., Yuan M.W., Dickens J.M., 1982, Dynamic analysis by direct superposition of Ritz vectors, Earthquake Engineering and Structural Dynamics, 10, 813-821

21. Zhou L., Zheng W.X., 2008, Three-dimensional analysis of thick plates by MLS-Ritz method, International Journal of Structural Stability and Dynamics, 8, 77-101 